\title{
Cavum septum pellucidum and cavum vergae with late-onset catatonia
}

\author{
Takehiko Yasaki, M.D, ${ }^{1}$ Yuka Takahashi, MD, ${ }^{1}$ Tohru Takahashi, MD, PhD, ${ }^{1}$ Shinsuke \\ Washizuka, MD, PhD, ${ }^{1}$ Naoji Amano, MD, PhD, ${ }^{1}$ Tokiji Hanihara, MD, $\mathrm{PhD}{ }^{1.2}$ \\ ${ }^{1}$ Department of Psychiatry, Shinshu University School of Medicine \\ ${ }^{2}$ School of Health Sciences, Shinshu University, Nagano, Japan
}

Corresponding author: Tokiji Hanihara MD, School of Health Science, Shinshu University Asahimachi 3-1-1, Matsumoto 390-8621, Japan

Phone: +81-263-37-2414

E-mail: qhaniha@shinshu-u.ac.jp 


\section{Abstract}

Objective: We report a case of late-onset catatonia associated with enlarged cavum septum pellucidum and cavum vergae.

Method: The patient (a 66-year-old woman) was initially treated with aripiprazole (12 $\mathrm{mg} /$ day) and lorazepam (1.5 mg/day). Lorazepam was subsequently increased to 3 $\mathrm{mg} /$ day, aripiprazole was tapered, and amoxapine $(50 \mathrm{mg} /$ day $)$ therapy was initiated. After 4 weeks, she was treated with electroconvulsive therapy (ECT).

Results: Following 8 ECT sessions, she began to ambulate normally and her mood appeared euthymic. By 10 treatments, echolalia vanished and catatonic behavior improved.

Conclusions: Developmental anomalies in the midline structure may increase susceptibility to psychosis, even in the elderly. 


\section{Introduction}

The septum pellucidum is a thin vertical membrane, with two laminae of both gray and white matter, which separates the lateral ventricles. During development, a space exists between the laminae, called the cavum septum pellucidum (CSP); this is filled with cerebrospinal fluid and usually disappears during infancy. However, CSP occasionally persists and may be associated with neurodevelopmental abnormalities of midline and limbic structures [1-3]. The cavum vergae (CV) is a normal variant of the septum pellucidum in which there is separation of the laminae with posterior extension to the splenium of the corpus callosum. Complete non-fusion of the two laminae of the septum pellucidum, a phenomenon termed combined CSP and CV, is the most extreme form of CSP. Associations between large CSP and functional psychosis disorders, especially schizophrenia, have been reported [1-5]. We present an unusual case of late-onset catatonia with CSP and CV.

\section{Case Report}

A 66-year-old housewife with a 2-year history of undifferentiated somatoform disorder was admitted to hospital presenting with altered mental status and stereotypic movement that had persisted for 3 weeks. She had no psychiatric illness or family history of psychosis. At 64 years, she had complained of vague physical symptoms, including toothache, thirstiness, difficulty in urination, and insomnia. At 66 years, she gradually 
became retarded and anxious. Occasionally, she repeated herself and experienced feelings of restlessness and choking. Brain magnetic resonance imaging (MRI) revealed mild brain atrophy; three small basilar artery aneurysms were incidentally found.

She was subsequently admitted to another hospital, where two of the three aneurysms were treated using endovascular coil embolization without complications. However, after discharge, she developed bizarre repetitive behavior; she compulsively turned her head or rolled on the floor. Gradually, she became withdrawn and was brought to our hospital. At admission, she was lying supine in the bed and staring at the ceiling; her blinking and spontaneous speech was markedly reduced, but pausing during speech was frequently observed. Echolalia was also noted. She experienced no delusions or hallucinations, but her mood seemed blunted. Paroxysmal movements, such as rolling of her upper extremities and tapping of her abdomen, were noted. There was no evidence of overt rigidity or gegenhalten. Her Mini Mental Status Examination score was 28/30. Electroencephalography showed no evidence of seizure activities; background activity was normal. MRI revealed mild frontal and parietal atrophy and enlarged CVP with CV (Fig. 1).

A diagnosis of retarded catatonia was suspected. She was subsequently treated with aripiprazole (12 mg/day) and lorazepam ( $1.5 \mathrm{mg} /$ day). Oral lorazepam was then increased to $3 \mathrm{mg} /$ day for 1 week, which induced partial relief of some symptoms; however, she remained withdrawn. Electroconvulsive therapy (ECT) was proposed, but her husband 
refused. After 4 weeks, she was still not adequately responding to medication; hence, aripiprazole was tapered and amoxapine $(50 \mathrm{mg} /$ day) therapy was initiated. Over the next month, she developed bizarre choreic movement in between periods of immobility. 99mTc-ethylcysteinate dimer single-photon emission computed tomography (SPECT) revealed slightly decreased perfusion in the bilateral temporo-occipital region. Upon her husband's consent, an ECT course was administered on the 80th hospital day. Bilateral stimulation was performed using a pulse wave ECT device (Thymatron System IV). After 8 treatments (3/week), she began normal ambulation and her mood appeared euthymic. By 10 treatments, the echolalia and mannerisms completely disappeared. An additional 10 ECT sessions were successfully completed; follow-up SPECT revealed no significant improvement in cerebral perfusion. She was then discharged.

\section{Discussion}

Catatonia is characterized by motor and behavioral abnormalities associated with changes in thought, mood, and vigilance. The most common symptoms are immobility, stupor, posturing, negativism, staring, waxy flexibility, and echophenomena [6,7]. Both benzodiazepines and ECT are effective treatment approaches [6].

Catatonia is associated with a wide variety of psychiatric disorders, as well as general medical conditions [6,7]. Historically, it has been classified as a subtype of schizophrenia, but is more commonly a consequence of mood disorders. Nevertheless, many patients 
experience idiopathic catatonia, i.e., catatonia not associated with any proposed diagnostic criteria for psychiatric or organic disorders [8]. In our case, the patient had no history of psychiatric illness; thus, her symptoms fit a diagnosis of idiopathic catatonia. However, she had a neurodevelopmental anomaly of CSP combined with CV.

Recent studies with high-resolution MRI have suggested that the presence of a small CSP is a normal variant of anatomy; however, a CSP $\geq 6 \mathrm{~mm}$ in size is considered abnormal. In fact, a large CSP can act as a marker for early developmental abnormalities associated with the corpus callosum and limbic systems $[1,3,4]$. To date, a large CSP is one of the most robust structural abnormality findings associated with a subgroup of schizophrenia $[4,5]$; however, the number of patients with schizophrenia and a combined large CSP and $\mathrm{CV}$ is relatively small $[4,9,10]$. Indeed, it is observed in less than $1 \%$ of all adults. To our knowledge, this is the first case report of a large CSP and CV in association with catatonia. While the incidence of a large CSP in catatonia is uncertain, there have been several reported cases of a large CSP in late-onset psychosis [11,12]. Several authors propose that psychosis may share, to some extent, neurodevelopmental abnormalities that involve midline structures $[3,4]$. Although anecdotal in nature, our findings suggest that developmental anomalies in midline structures may increase the susceptibility of certain individuals, even the elderly, to psychosis. 


\section{References}

[1] Nopoulos P, Swayze V, Flaum M, et al. Cavum septi pellucidi in normal and patients with schizophrenia as detected by magnetic resonance imaging. Biol Psychiatry 1996;741:1102-8.

[2] Rajarethinam R, Miedler J, DeQuardo J, et al. Prevalence of cavum septum pellucidum in schizophrenia studied with MRI. Schizophr Res 2001;48:201-5.

[3] Kasai K, McCarley RW, Salisbury DF, et al. Cavum septi pellucidi in first-episode schizophrenia and first-episode affective psychosis: an MRI study. Schizophr Res 2004;71:65-76.

[4] Trzesniak C, Oliveira IR, Kempton MJ, et al. Are cavum septum pellucidum abnormalities more common in schizophrenia spectrum disorders? A systematic review and meta-analysis. Schizophr Res 2011;125:1-12.

[5] Shenton ME, Dickey CC, Frumin M, McCarley RW. A review of MRI findings in schizophrenia. Schizophr Res 2001;49:1-52.

[6] Rosebush PI, Mazurek MF. Catatonia and its treatment. Schizophr Bull 2010;36:239-42.

[7] Fink M, Taylor MA. The catatonia syndrome: forgotten but not gone. Arch Gen Psychiatry 2009;66:1173-7.

[8] Benegal V, Hingorani S, Khanna S. Idiopathic catatonia: validity of the concept. 
Psychopathology 1993;26:41-6.

[9] Nopoulos P, Krie A, Andreasen, NC. Enlarged cavum septi pellucidi in patients with schizophrenia: clinical and cognitive correlates. J Neuropsychiatry Clin Neurosci 2000:12:344-9.

[10] Wolf SS, Hyde TM, Weinberger DR. Malformations of the septum pellucidum: two distinctive cases in association with schizophrenia. J Psychiatry Neurosci 1994;19:140-4. [11] Shrestha B. Late onset of psychotic symptoms in a patient with cavum septum pellucidum and cavum vergae. J Neuropsychiatry Clin Neurosci 2012;24:e43-4.

[12] Tsujino N, Nemoto T, Yamaguchi $T$, et al. Cerebral blood flow changes in very-late-onset schizophrenia-like psychosis with catatonia before and after successful treatment. Psychiatry Clin Neurosci 2011;65:600-3. 


\section{Figure legend}

Fig. 1

T1-weighted axial image showing enlargement of lateral ventricles with presence of cavum septum pellucidum and cavum vergae.

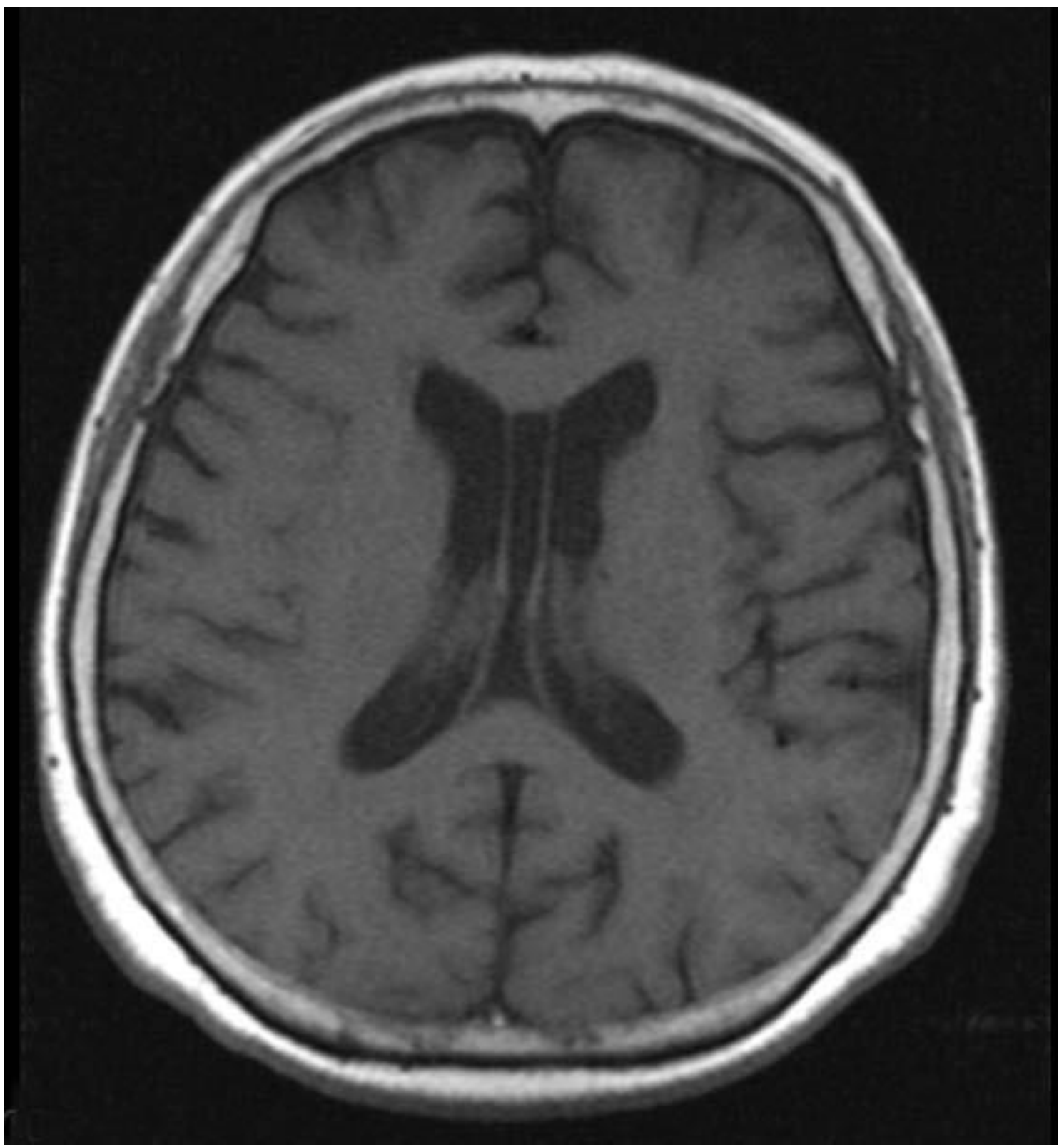

